\title{
TITLE:
}

\section{Hybrid dynamical system as model for cascading outage in a power system}

\section{AUTHOR(S):}

Susuki, Yoshihiko; Takatsuji, Yu; Hikihara, Takashi

\section{CITATION:}

Susuki, Yoshihiko ... [et al]. Hybrid dynamical system as model for cascading outage in a power system. 40th North American Power Symposium (NAPS2008) 2008: 5307356.

ISSUE DATE:

2008-09

URL:

http://hdl.handle.net/2433/66189

RIGHT: 


\title{
Hybrid Dynamical System as Model for Cascading Outage in a Power System
}

\author{
Yoshihiko Susuki, Yu Takatsuji, Takashi Hikihara \\ Kyoto University, Department of Electrical Engineering, Katsura, Nishikyo, Kyoto 615-8510 Japan \\ susuki@ieee.org
}

\begin{abstract}
This paper uses hybrid dynamical systems as a mathematical model for cascading outages in a power system. Hybrid dynamical systems can combine families of flows describing swing dynamics of generators with switching rules describing protection control mechanisms. We refer to data observed in a cascading outage in the September 2003 blackout in Italy and show a hybrid dynamical system by which propagation of outages reproduced is consistent with the data.
\end{abstract}

\section{INTRODUCTION}

Complex dynamics have recently emerged as a major issue in electric power systems [1], [2]. Cascading outage is one important example of complex dynamics and is the generic mechanism by which outages propagate to cause widespread blackouts of power systems [3], for example, the August 2003 blackout in North America [4]. Understanding such complex dynamics is required for preventing them, in particular, minimizing damage caused by blackouts, and for making a robust infrastructure with electricity supply.

Transient stability of power systems is closely related to occurrence of cascading outages. Transient stability is concerned with the ability of power system to maintain synchronism when subjected to a severe disturbance [5], [6]. The stability is mainly governed by swing dynamics of synchronous machines. The swing dynamics usually cause overcurrents and heavy power flows. This often results in outages of transmission and generation facilities by relay operation to protect them. This cascading trend continues in the worst cases. The interaction between swing dynamics governing transient stability and protection operation is hence one mechanism of cascading outages, for example, the September 2003 blackout in Italy [7], [4]. As indicated by [8], [9], [10], cascading outage contains hybrid nature, implying that it is modeled with both continuous- and discrete-valued variables.

The purpose of this paper is to report a mathematical model for cascading outage in a power system. Following the importance of transient stability, we treat the interaction between swing dynamics and protection operation. The model reported here is a hybrid dynamical system [11], [12], [13] and consists of families of flows describing swing dynamics and their switching rules that are based on protection control mechanisms. We refer to data on a cascading outage in the September 2003 blackout in Italy [7] and show a hybrid dynamical model by which propagation of outages reproduced is consistent with the data. Note that the contents of this paper have been submitted to archival journal [14].

\section{Cascading Outage in the September 2003 BLACKOUT IN ITALY}

Before model development in Secs. III and IV, this section reviews data observed in a cascading outage in the September 2003 Blackout in Italy. The following review is based on [7]. The Italian power system experienced a large blackout on September 28, 2003. The blackout affected an area with an estimated 60 million people and load variation in the continental grid from about $24000 \mathrm{MW}$ at the early hours of the day, up to $50000 \mathrm{MW}$ in the mid-day. This is regarded as the largest blackout ever to happen in Italy.

Figure 1 shows (a) $400 \mathrm{kV}$ and (b) $220 \mathrm{kV}$ power transmission networks interconnecting Italy (ITALIE therein) with France and Switzerland (SUISSE). A cascading outage or trip of transmission lines is indicated according to the time they tripped, starting from \#1 at 3:01.42 up to \#9 at 3:25.33. The cascading trip resulted in the separation of Italian network from Switzerland and French ones and, after further cascading trips of generators in Italy, finally led to the blackout.

Figure 1(c) shows frequency traces in North Italy at Piossasco, S. Rocco, and Musignano EHV substations from 3:25.12 up to $3: 26.12$. Three pulsing behaviors are observed in Fig. 1(c) during the period from line trip \#2 at 3:25.21 up to \#5 at 3:25.27. It is indicated in [7] that the observed behaviors are caused by the interaction between transient stability governed by swing dynamics and protection operation. The swing dynamics cause heavy power flows that trigger the protection operation. The operation is normally based on overcurrent or distance relaying. We treat the swing dynamics related to the cascading trip of transmission lines.

It should be noted that various frequency dynamics are observed in Fig. 1(c). For example, the line trip \# 8 at 3:25.33 in Figs. 1(a) and (b) triggered continuous decrease of frequency below $48.5 \mathrm{~Hz}$ in Fig. 1(c). The associated dynamics result in the isolation of Italian network and the blackout. Here, from phenomenological point of view, they are modeled through an interacting system with swing dynamics, voltage dynamics, and protection operation. However, this modeling and analytical study are not easy. As a first step of analytical study on cascading outage, in this paper we focus on the relatively simple cascading outage, and the detailed modeling is in future work. 

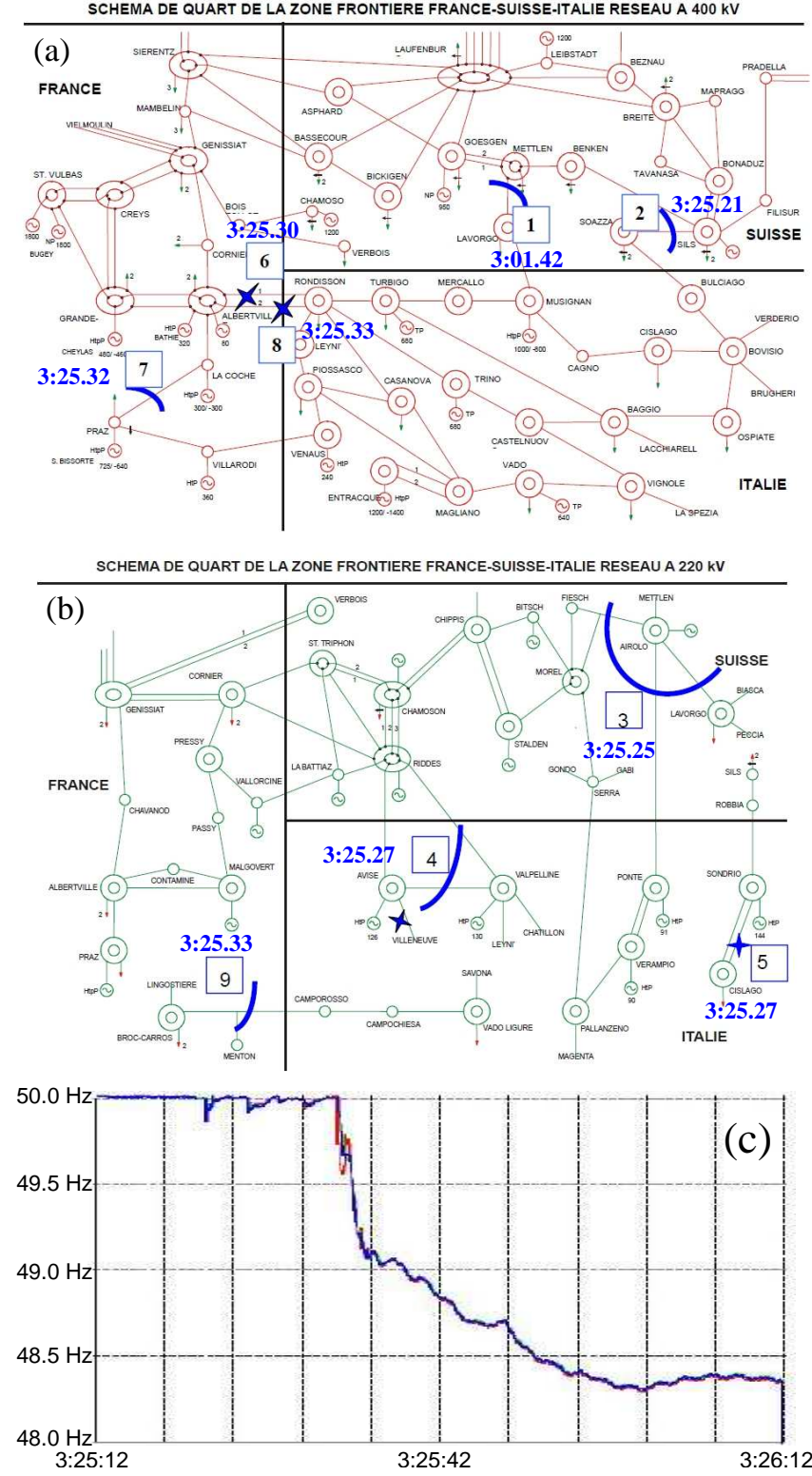

Fig. 1. Data of the September 2003 Blackout in Italy. Adapted and reprinted with permission from [7] (C)2004 IEEE.

\section{Hybrid Model for the CASCAding OUtAge}

This section derives a hybrid dynamical system as a mathematical model for the cascading outage in Sec. II.

\section{A. Six-Machine Power System Model}

Figure 2 shows six-machine power system model. The interconnected power system in Figs. 1(a) and (b) is decomposed into several subsystems that are represented by equivalent synchronous machines and constant power loads. Note that the decomposition is not unique and depends on modeling purpose. The six equivalent machines G1-G6 represent the Switzerland and French power systems, and the loads, denoted by the arrows, represents the Italian power system. The direction of power flows is hence from the Switzerland and

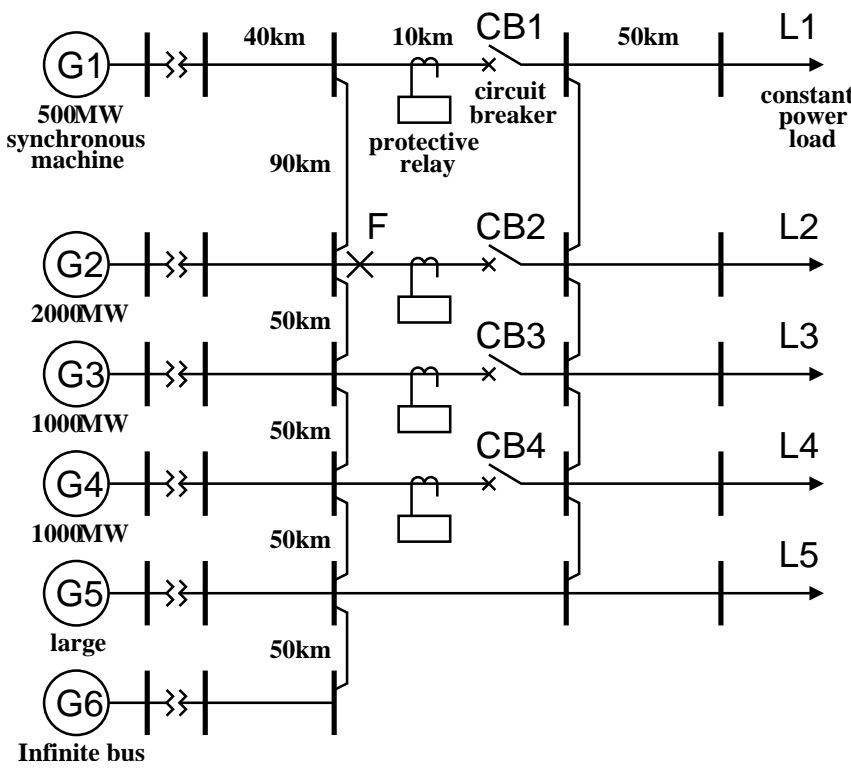

Fig. 2. Six-machine power system model that is based on Figs. 1(a) and (b)

French power systems to the Italian one. This is consistent with the data in [7]. G6 is assumed to be the infinite bus [5]. The transmission lines with overcurrent relays and circuit breakers $\mathrm{CB} 1-\mathrm{CB} 4$ connects the Italian system with others. CB1 is related to the line trip \#5 in Fig. 1(b), CB2 to \#2 in Fig. 1(a), CB3 to \#3 in Fig. 1(b), and CB4 to \#4 in Fig. 1(b). Hence the propagation of line trips in the data is $\mathrm{CB} 2 \rightarrow \mathrm{CB} 3 \rightarrow \mathrm{CB} 4 \rightarrow \mathrm{CB} 1$ under swing dynamics of $\mathrm{G} 1-$ G5 in Fig. 2. The following subsections treat the interaction between the swing dynamics and protection operation in Fig. 2.

\section{B. Continuous-Time Model for Swing Dynamics}

We use the so-called classical model [15], [16] of swing dynamics of synchronous machines. The classical model describes swing dynamics of machine $\mathrm{G} i(i=1, \ldots, 5)$ by the following ordinary differential equations:

$$
\left.\begin{array}{rl}
\dot{\delta}_{i} & =\omega_{i}, \\
\frac{P_{\mathrm{r} i}}{P_{\mathrm{b}}} \frac{H_{i}}{\pi f_{\mathrm{s}}} \dot{\omega}_{i} & =p_{\mathrm{m} i}-d_{i} \omega_{i}-p_{\mathrm{e} i}\left(\delta_{1}, \ldots, \delta_{6}\right) .
\end{array}\right\}
$$

$\dot{\delta}_{i}$ is the time $(t)$ differentiation of $\delta_{i} . \delta_{i}$ is the rotor angle position of $\mathrm{G} i$ with respect to the infinite bus $\mathrm{G} 6, \omega_{i}$ the rotor speed deviation of $\mathrm{G} i$ relative to system angular frequency $2 \pi f_{\mathrm{s}} . \delta_{6}$ for $\mathrm{G} 6$ is assumed to be zero. $P_{\mathrm{b}}, P_{\mathrm{r} i}, H_{i}, f_{\mathrm{s}}, p_{\mathrm{m} i}$, and $d_{i}$ are parameters of the classical model (1). $P_{\mathrm{b}}$ is the base power, $P_{\mathrm{r} i}$ the machine rating of $\mathrm{G} i$, and $H_{i}$ its per-unit inertia constant. $p_{\mathrm{m} i}$ is the mechanical input power to $\mathrm{G} i$, and $d_{i}$ its damping coefficient. $p_{\mathrm{e} i}$ stands for the electrical output power of $\mathrm{G} i$ and is given by the following function of all rotor 


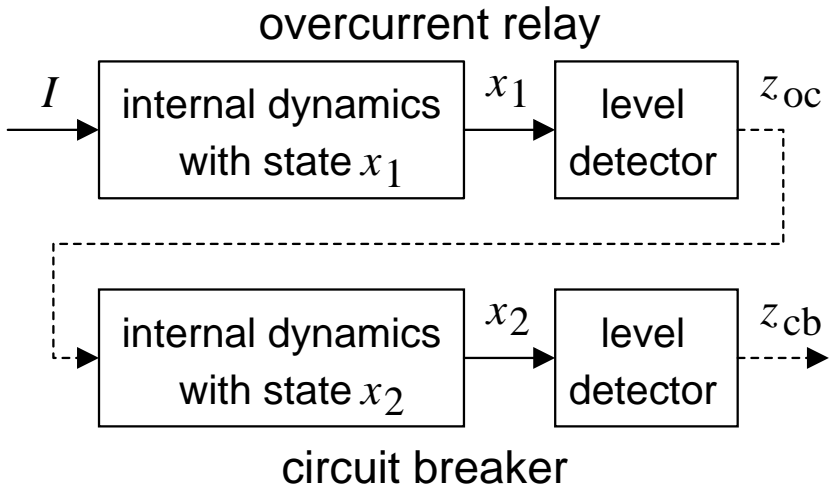

Fig. 3. Control mechanisms of induction-disc type overcurrent relay and circuit breaker in [17]

angle positions $\delta_{1}, \ldots, \delta_{6}$ :

$$
\begin{aligned}
p_{\mathrm{e} i} \triangleq & G_{i i} E_{i}^{2}+\sum_{j=1, j \neq i}^{6} E_{i} E_{j}\left\{G_{i j} \cos \left(\delta_{i}-\delta_{j}\right)\right. \\
& \left.+B_{i j} \sin \left(\delta_{i}-\delta_{j}\right)\right\} .
\end{aligned}
$$

$E_{i}$ is the terminal voltage of $\mathrm{G} i, G_{i i}$ its internal conductance, and $G_{i j}+\mathrm{j} B_{i j}$ the transfer admittance between $\mathrm{G} i$ and $\mathrm{G} j$. Note that the loads in Fig. 2 are modeled as passive impedance [16]. $E_{i}, G_{i i}, G_{i j}$, and $B_{i j}$ are also the parameters and can be determined from power flow calculation.

\section{Control Mechanisms of Protection System}

We next describe control mechanisms of a protection system with overcurrent relays and circuit breakers. Perez et al. [17] modeled protective relay systems for power system dynamics analysis. Our modeling relies on their mathematical models of relay systems.

Figure 3 shows control mechanisms of induction-disc type overcurrent relay and circuit breaker. The solid arrows denote continuous signals, and the broken arrows discrete ones. The relay and breaker have internal dynamics represented by ordinary differential equations of state variables $x_{1}$ for relay and $x_{2}$ for circuit breaker. $I$ is the input of line current and is a function of rotor angle positions $\delta_{1}, \ldots, \delta_{6}$. Level detector produces a discrete output 1 (or 0 ) when the magnitude of input $x_{1}$ or $x_{2}$ is greater (or less) than a prescribed threshold. $z_{\mathrm{oc}}$ and $z_{\mathrm{cb}}$ in Fig. 3 denote the discrete outputs of overcurrent relay and circuit breaker. The condition $z_{\mathrm{cb}}=1$ is associated with that when the corresponding circuit breaker opens (trips), in other words, the line is disconnected; $z_{\mathrm{cb}}=0$ when it closes. Thereby the output $z_{\mathrm{cb}}$ determines the condition of transmission lines and regulates the network topology of sixmachine power system model.

The internal dynamics and level detector of induction-disc type overcurrent relay are described in [17] as

$$
\left.\begin{array}{rl}
\dot{x}_{1} & =f(I)\left\{1-\nu\left(x_{1}-x_{\mathrm{TDS}}\right) g(I)\right\}, \\
z_{\mathrm{oc}} & =\nu\left(x_{1}-x_{\mathrm{TDS}}\right),
\end{array}\right\}
$$

where

$$
\begin{aligned}
& f(I) \triangleq K\left\{\left(\frac{I}{I_{\mathrm{TAP}}}\right)^{2}-1\right\}, \\
& g(I) \triangleq \nu\left(I-I_{\mathrm{TAP}}\right), \\
& \nu(x) \triangleq\left\{\begin{array}{ll}
0 & \text { if } x \leq 0, \\
1 & \text { if } x>0 .
\end{array}\right\}
\end{aligned}
$$

The level detector in Fig. 3 is defined as the step function $\nu$ of state $x_{1} . I_{\mathrm{TAP}}, x_{\mathrm{TDS}}$, and $K$ are the tuning parameters of overcurrent relay. $I_{\mathrm{TAP}}$ is the prescribed threshold value of input current $I, x_{\operatorname{TDS}}$ the threshold value of state $x_{1}$, and $K$ is the acceleration factor of internal dynamics. On the other hand, the internal dynamics and level detector of circuit breaker are described in [17] as

$$
\left.\begin{array}{rl}
\tau \dot{x}_{2} & =z_{\mathrm{oc}}\left\{1-\nu\left(x_{2}-x_{\mathrm{tr}}\right)\right\} \\
z_{\mathrm{cb}} & =\nu\left(x_{2}-x_{\mathrm{tr}}\right) .
\end{array}\right\}
$$

The parameters $x_{\mathrm{tr}}$ and $\tau$ for operation of the circuit breaker are also assumed to be constant. $x_{\mathrm{tr}}$ is the threshold value of $x_{2}$, and $\tau$ the time constant for internal dynamics.

\section{Hybrid Dynamical System}

By combining the continuous-time model for swing dynamics in Sec. III-B with the models of protection operation in Sec. III-C, we can derive a hybrid dynamical system as a mathematical model for the cascading outage. A definition of hybrid dynamical systems is given in [11], [12], [13] that we use in what follows.

First, all candidates of network topologies of the sixmachine power system model are described using a finite index set. This section aims to model the interaction between swing dynamics of G1-G5 and line trips of CB1-CB4. The parameters $G_{i i}, G_{i j}$, and $B_{i j}$ in the classical model (1) discontinuously change when the circuit breakers trip and disconnect the lines. Then it is needed to describe all candidates of network topologies explicitly. A finite index set $Q$ is defined as

$$
\begin{aligned}
Q \triangleq & \{1234,123,124,134,234, \\
& 12,13,14,23,24,34,1,2,3,4,0\} .
\end{aligned}
$$

The index $1234 \in Q$ denotes the network topology under no trip of CB1-CB4, 123 under trip of CB4, 0 under all trips of CB1-CB4, and so on.

Second, the swing dynamics of G1-G5 are described using a family of flows. To do so, we re-write the classical model (1) that makes it possible to take the network topologies into account. The electrical output $p_{\mathrm{e} i}^{(\alpha)}$ indexed by $\alpha \in Q$ that describes one network topology is given by

$$
\begin{aligned}
p_{\mathrm{e} i}^{(\alpha)}= & G_{i i}^{(\alpha)} E_{i}^{2}+\sum_{j=1, j \neq i}^{6} E_{i} E_{j}\left\{G_{i j}^{(\alpha)} \cos \left(\delta_{i}-\delta_{j}\right)\right. \\
& \left.+B_{i j}^{(\alpha)} \sin \left(\delta_{i}-\delta_{j}\right)\right\} .
\end{aligned}
$$

The parameter $G_{i i}^{(\alpha)}$ denotes the internal impedance of $\mathrm{G} i$ for index $\alpha$, and similarly for $G_{i j}^{(\alpha)}$ and $B_{i j}^{(\alpha)}$. Here $V_{\alpha}$ denotes an 
open subspace of $S^{5} \times \mathbb{R}^{5}$ whose elements are the rotor angle positions $\delta_{1}, \ldots, \delta_{5}$ and rotor speed deviation $\omega_{1}, \ldots, \omega_{5}$. The subspace is called chart [11], [13]. The collection of charts, $V=\bigcup_{\alpha \in Q} V_{\alpha}$, is also called atlas [11], [13]. Each chart has associated with it a flow $\mathcal{F}_{\alpha}: V_{\alpha} \rightarrow \mathbb{R}^{10}$ described by the classical model (1) indexed by $\alpha \in Q$. Thus the swing dynamics of G1-G5 are described by a family of flows, $\left\{\mathcal{F}_{\alpha}\right\}_{\alpha \in Q}$, defined on the atlas $V$.

Third, the protection control mechanisms are described by switching between flows. For the control mechanisms in Sec. III-C, $V_{c}$ is defined as an open subspace of $\mathbb{R}^{8}$ whose elements are the internal states $\left(x_{11}, x_{21}, \ldots, x_{14}, x_{24}\right)^{\mathrm{T}}$. The pair $\left(x_{1 i}, x_{2 i}\right)$ represent the states for $\mathrm{CB} i$. The state equations (3) and (5) define a flow $\mathcal{F}_{\mathrm{c}}: V_{\alpha} \times V_{\mathrm{c}} \rightarrow \mathbb{R}^{8}$. The reason that $V_{\alpha}$ is here contained in the definition of $\mathcal{F}_{\mathrm{c}}$ is that equation (3) has the input current $I$ which is a function of rotor angle positions $\left(\delta_{1}, \ldots, \delta_{5}\right) \in V_{\alpha}$. Then, by referring to [13], for each $\alpha \in Q$ we have a collection of functions $h_{\alpha}^{\beta}: V_{\alpha} \times V_{\mathrm{c}} \rightarrow\{0,1\}$ indexed by $\beta$. As points flow across a chart, the threshold functions $h_{\alpha}^{\beta}$ monitor whether a transition to a (possibly) new chart should occur at that instant. When the function $h_{\alpha}^{\beta}$ becomes unity, the trajectory switches to a chart described by the index $\beta$. The concrete description of $h_{\alpha}^{\beta}$ is given by the discrete outputs $z_{\mathrm{cb} 1}, \ldots, z_{\mathrm{cb} 4}$ in equation (5). Switching between charts occurs via mappings $\mathcal{T}_{\alpha}^{\beta}$ with domains in $V_{\alpha}$ and ranges in $V_{\beta}$. It is assumed in our modeling that there is no reset of variables, in other words, $\mathcal{T}_{\alpha}^{\beta}$ is the identity mapping.

Thus, we can describe the interaction between swing dynamics and protection operation using a hybrid dynamical system as the following collection $\mathrm{H}$ :

$$
\mathrm{H}=\left(Q, V_{\alpha}, \mathcal{F}_{\alpha}, h_{\alpha}^{\beta}, \mathcal{T}_{\alpha}^{\beta}, V_{\mathrm{c}}, \mathcal{F}_{\mathrm{c}}\right) .
$$

Here it is worth mentioning that $\mathrm{H}$ is re-formulated as a form of hybrid automaton [18], [19]. $Q$ is the set of finite discrete states, and $V_{\alpha} \times V_{\mathrm{c}}$ the set of continuous states. $\mathcal{F}_{\alpha}$ and $\mathcal{F}_{\mathrm{c}}$ represent the continuous dynamics (vector field) described by equations (1), (3) and (5), and $\mathcal{T}_{\alpha}^{\beta}$ the reset function [19] or, in our modeling, mapping between different discrete states. $h_{\alpha}^{\beta}$ is regarded as a guard condition [19] for discrete evolution that determines whether mapping $\mathcal{T}_{\alpha}^{\beta}$ should occur. Verification algorithms and tools for hybrid automaton are hence applicable to our hybrid model for the cascading outage of power system.

\section{Analysis of the Cascading Outage}

This section analyzes the hybrid dynamical system $\mathrm{H}$. Table I shows configuration and parameter setting in sixmachine power system model which are used for numerical simulations. They are based on the data in [7]. Here it is not possible to find in the literature all parameters required by our hybrid model. The parameters have been chosen to match the data in [7]. It is supposed that there is no control equipment of synchronous machines such as AVR (Automatic Voltage Regulator) and PSS (Power System Stabilizer), and that there is also no LRT (Load Ratio control Transformer)
TABLE I

CONFIGURATION AND PARAMETER SETTING OF SIX-MACHINE POWER SYSTEM MODEL

\begin{tabular}{|c|c|c|}
\hline VA base $P_{\mathrm{b}}$ & & $2000 \mathrm{MW}$ \\
\hline Voltage base & & $400 \mathrm{kV}$ \\
\hline System frequency $f_{\mathrm{s}}$ & & $50 \mathrm{~Hz}$ \\
\hline \multirow[t]{4}{*}{ Machine rating $P_{\mathrm{r} i}$ of $\mathrm{G} i$} & $i=1$ & $500 \mathrm{MW}$ \\
\hline & $i=2$ & $2000 \mathrm{MW}$ \\
\hline & $i=3,4$ & $1000 \mathrm{MW}$ \\
\hline & $i=5$ & $200 \mathrm{GW}$ \\
\hline $\begin{array}{l}\text { Per-unit inertia constant } H_{i} \\
\text { of } \mathrm{G} i\end{array}$ & $i=1, \ldots, 5$ & $5 \mathrm{~s}$ \\
\hline Damping coefficient $d_{i}$ of $\mathrm{G} i$ & $i=1, \ldots, 5$ & 0.05 \\
\hline \multirow{5}{*}{$\begin{array}{l}\text { Mechanical input power } p_{\mathrm{m} i} \\
\text { to } \mathrm{G} i\end{array}$} & $i=1$ & 0.2 \\
\hline & $i=2$ & 0.95 \\
\hline & $i=3$ & 0.45 \\
\hline & $i=4$ & 0.35 \\
\hline & $i=5$ & 1.25 \\
\hline Terminal voltage $E_{i}$ of $\mathrm{G} i$ & $i=1, \ldots, 5$ & 1 \\
\hline $\begin{array}{l}\text { Line inductance } \\
\text { of } \pi \text {-equivalent circuit model }\end{array}$ & & $0.9 \mathrm{mH} / \mathrm{km}$ \\
\hline Line resistance & & $0 \Omega / \mathrm{km}$ \\
\hline Line capacitance & & $0 \mathrm{~F} / \mathrm{km}$ \\
\hline Transformer impedance & & 0.15 \\
\hline \multirow{5}{*}{$\begin{array}{l}\text { Constant active power flow } \\
\text { to load } L i\end{array}$} & $i=1$ & 0.2 \\
\hline & $i=2$ & 0.95 \\
\hline & $i=3$ & 0.45 \\
\hline & $i=4$ & 0.35 \\
\hline & $i=5$ & 1.25 \\
\hline $\begin{array}{l}\text { Constant reactive power flow } \\
\text { to load } L i\end{array}$ & $i=1, \ldots, 5$ & 0 \\
\hline \multirow{3}{*}{$\begin{array}{l}\text { Parameters of } \\
\text { overcurrent relay }\end{array}$} & $I_{\mathrm{TAP}}$ & 1.03 \\
\hline & $K$ & 16 \\
\hline & $x_{\mathrm{TDS}}$ & 1 \\
\hline \multirow[t]{2}{*}{ circuit breaker } & $\tau$ & $0.1 \mathrm{~s}$ \\
\hline & $x_{\mathrm{tr}}$ & 1 \\
\hline
\end{tabular}

with machine buses. By numerical simulations we demonstrate that the hybrid dynamical system reproduces the propagation of outages in Fig. 1 and provides a dynamical feature of the propagation.

\section{A. Numerical Experiment}

We perform numerical simulations for swing dynamics and protection operation in six-machine power system model. The bus voltages are fixed through power flow calculation. Steady state conditions of rotor angle position $\delta_{i}(i=1, \ldots, 5)$ are also determined through the calculation which are used as the initial condition $\delta_{i}(t=0 \mathrm{~s})$. By putting the initial condition $\omega_{i}(0 \mathrm{~s})=0(i=1, \ldots, 5)$, it is possible to start numerical simulations from the steady state condition. The initial conditions of internal states $\left(x_{1 i}, x_{2 i}\right)(i=1, \ldots, 4)$ are also fixed at $\left(x_{1 i}(0 \mathrm{~s}), x_{2 i}(0 \mathrm{~s})\right)=(0,0)$. The parameters $G_{i i}^{(\alpha)}, G_{i j}^{(\alpha)}$, and $B_{i j}^{(\alpha)}$ for each $\alpha \in Q$ are also determined through the power flow calculation. It is here supposed that the system model is in the steady state condition at $t<0 \mathrm{~s}$, that a line-to-ground fault occurs at point $\mathrm{F}$ near G2 bus at $t=0 \mathrm{~s}$, and that the fault is removed at $t=t_{\mathrm{c}} \cdot t_{\mathrm{c}}$ corresponds to the fault duration.

Figure 4 shows time responses of rotor angle position $\delta_{i}$, rotor speed deviation $\omega_{i}$, internal states $\left(x_{1 i}, x_{2 i}\right)$ and discrete outputs $\left(z_{\mathrm{oc} i}, z_{\mathrm{cb} i}\right)$ for protection operation. The figure is 

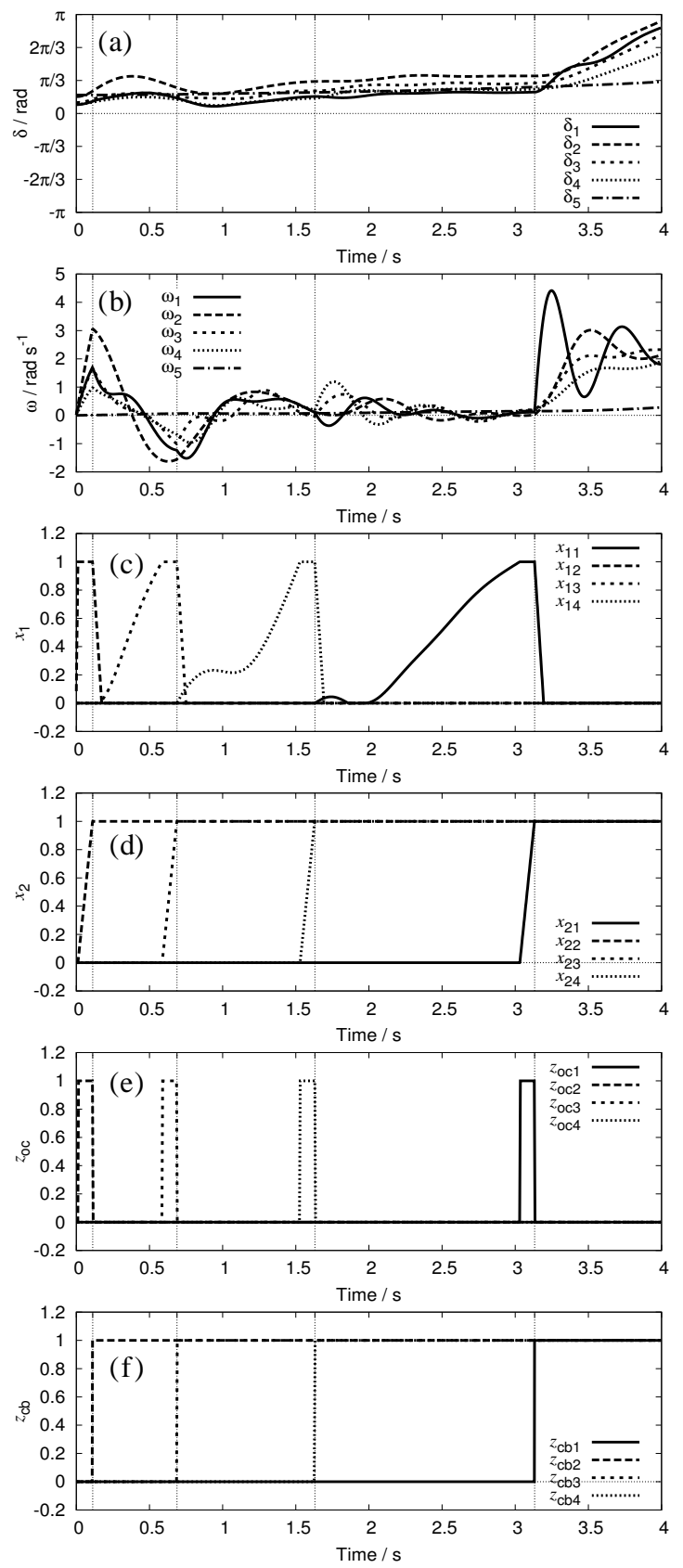

Fig. 4. Swing dynamics and protection operation in six-machine power system model. (a) Rotor angle position $\delta_{i}$; (b) rotor speed deviation $\omega_{i}$; (c) internal state $x_{1 i}$ of overcurrent relays; (d) internal state $x_{2 i}$ of circuit breakers; (e) discrete output $x_{\mathrm{oc} i}$ of overcurrent relays; (f) discrete output $z_{\mathrm{cb} i}$ of circuit breakers.

for fault duration $t_{\mathrm{c}}=0.112 \mathrm{~s} \sim 5.6$ cycles of a $50-\mathrm{Hz}$ sine wave. A cascade of line trips is observed: CB2 $(t=$ $0.112 \mathrm{~s}) \rightarrow \mathrm{CB} 3(0.688 \mathrm{~s}) \rightarrow \mathrm{CB} 4(1.631 \mathrm{~s}) \rightarrow \mathrm{CB} 1(3.133 \mathrm{~s})$. The lines CB1-CB4 do not exist in the system model, and finally all the machines lose synchronism with the infinite bus. This reproduced propagation of outages is consistent with the data in Sec. II. The occurrence of propagation is dependent on the fault duration $t_{\mathrm{c}}$. This dependency is partly analyzed
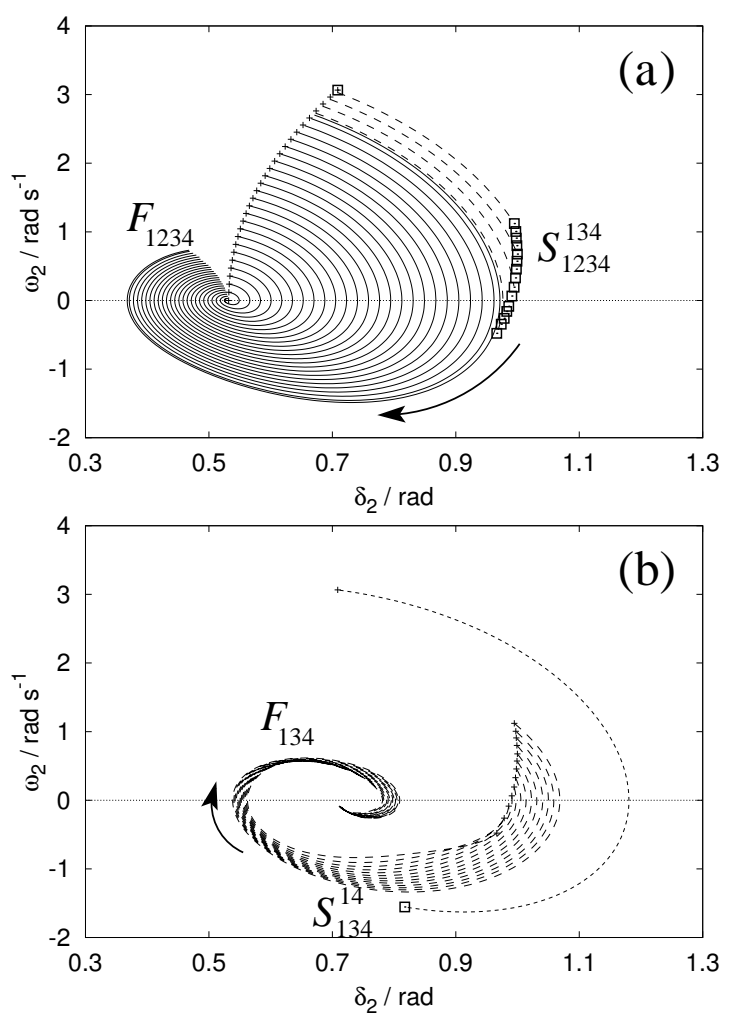

Fig. 5. Switching of flows governing the cascading outage in Fig. 4. (a) $\mathcal{F}_{1234}$ and (b) $\mathcal{F}_{134}$.

in the next subsection. On the other hand, the numerical swings for rotor speed deviation $\omega_{i}$ in Fig. 4 are at most $3 \mathrm{rad} / \mathrm{s} \sim 0.5 \mathrm{~Hz} \times 2 \pi$ and are not consistent with the data. This is because the data in Fig. 2 is measured at the large machine G5, and the places where it is measured in Fig. 1 are different from machines G1-G4 in Fig. 2.

\section{B. A Dynamical Study}

Sequential portraits of the flow described by the classical model (1) are presented. Since the flows are defined on the atlas $V$ with high dimensions, it is hard to visualize all the flow components. We hence focus on the flow that is obtained by varying the fault duration $t_{\mathrm{c}}$ in a given range $[0 \mathrm{~s}, T]$. $T$ corresponds to the maximum duration.

Figure 5 shows trajectories of the hybrid dynamical system for $T=0.112 \mathrm{~s}$. The trajectories are obtained by numerical integration of the classical model (1) for several values of $t_{\mathrm{c}}$ in $[0 \mathrm{~s}, T]$ and are projected onto $\delta_{2}-\omega_{2}$ plane. The right arc starting from $\left(\delta_{2}, \omega_{2}\right) \sim(0.52,0)$ shows the initial conditions on flow $\mathcal{F}_{1234} . \mathcal{F}_{\alpha}$ denotes the flow described by the classical model (1) indexed by $\alpha$, and $S_{\alpha}^{\beta}$ the switching points at which the system's state is transferred from $\mathcal{F}_{\alpha}$ to $\mathcal{F}_{\beta}$. The trajectories, denoted by the solid lines in Fig. 5, contain no switching of flows and converge to a stable equilibrium point on $\mathcal{F}_{1234}$. On the other hand, the trajectories, denoted by the dotted lines in Fig. 5, contain at least one switching of flows. After the system's state is transferred from $\mathcal{F}_{1234}$ to 
$\mathcal{F}_{134}$, most of the trajectories converge to a stable equilibrium point on $\mathcal{F}_{134}$. Here there is one trajectory in Fig. 5 for which the system's state is further transferred from $\mathcal{F}_{134}$, to $\mathcal{F}_{14}$, and to $\mathcal{F}_{1}$. The sequence of switching points is $S_{1234}^{134} \rightarrow S_{134}^{14} \rightarrow S_{14}^{1} \rightarrow S_{1}^{0}$. The trajectory finally diverges on flow $\mathcal{F}_{0}$ and corresponds to the cascading outage shown in Fig. 4.

Hence it is said that the cascading outage in Fig. 4 is produced as a result of the interplay between the continuous flows describing swing dynamics of synchronous machines and their switching rules describing protection control mechanisms. The details of the dynamical analysis are presented in [14].

\section{CONCLUSION}

We reported a hybrid dynamical system as a mathematical model for cascading outage in a power system. The model consists of a family of flows describing swing dynamics of synchronous machines and their switching rules describing control mechanisms of protection operation. This paper referred to data on a cascading outage in the September 2003 blackout in Italy and provided a hybrid dynamical system by which propagation of outages reproduced was consistent with the data. Analysis of hybrid dynamical system in Sec. IV indicates that switching of flows produces the cascading outage. The contribution of this paper is the derivation and analysis of hybrid dynamical system as model for cascading outage in a real power system.

\section{ACKNOWLEDGMENT}

We thank Dr. Yueheng Lan (University of California, Santa Barbara) for his careful reading of the manuscript.

\section{REFERENCES}

[1] P. Fairley, "The unruly power grid," IEEE Spectrum, vol. 41, no. 8, pp. 22-27, August 2004.

[2] C. W. Gellings and K. E. Yeager, "Transforming the electric infrastructure," Physics Today, vol. 57, no. 12, pp. 45-51, December 2004.

[3] I. Dobson, B. A. Carreras, V. E. Lynch, and D. E. Newman, "Complex systems analysis of series of blackouts: Cascading failure, critical points, and self-organization," CHAOS, vol. 17, no. 2, p. 02610, June 2007.

[4] G. Andersson, P. Donalek, R. Farmer, N. Hatziargyriou, I. Kamwa, P. Kundur, N. Martins, J. Paserba, P. Pourbeik, J. Sanchez-Gasca, R. Schulz, A. Stankovic, C. Taylor, and V. Vittal, "Causes of the 2003 major grid blackouts in North America and Europe, and recommended means to improve system dynamic performance," IEEE Transactions on Power Systems, vol. 20, no. 4, pp. 1922-1928, November 2005.

[5] E. W. Kimbark, Power System Stability. New York: John Wiley \& Sons, 1947, vol. I.

[6] H. D. Chiang, "Power system stability," in Wiley Encyclopedia of Electrical and Electronics Engineering, J. G. Webster, Ed. New York: John Wiley \& Sons, March 1999, pp. 105-137.

[7] S. Corsi and C. Sabelli, "General blackout in Italy Sunday September 28, 2003, h. 03:28:00," in Proceedings of the IEEE PES General Meeting, vol. 2, Denver, USA, June 2004, pp. 1691-1702.

[8] W. H. Esselman, D. J. Sobajic, and J. Maulbetsch, "Hybrid discrete and continuous control for power systems," Discrete Event Dynamic Systems: Theory and Applications, vol. 9, pp. 297-318, 1999.

[9] L. H. Fink, "Discrete events in power systems," Discrete Event Dynamic Systems: Theory and Applications, vol. 9, pp. 319-330, 1999.

[10] I. A. Hiskens and M. A. Pai, "Hybrid systems view of power system modeling," in Proceedings of the IEEE International Symposium on Circuits and Systems, vol. II, Geneva, Switzerland, May 2000, pp. 228 231.
[11] A. Back, J. Guckenheimer, and M. Myers, "A dynamical simulation facility for hybrid systems," in Hybrid Systems, ser. Lecture Notes in Computer Science 736, R. L. Grossman, A. P. Ravn, and H. Rischel, Eds. Springer-Verlag, 1993, pp. 255-267.

[12] S. D. Johnson, "Simple hybrid systems," International Journal of Bifurcation and Chaos, vol. 4, no. 6, pp. 1655-1665, June 1994.

[13] J. Guckenheimer and S. Johnson, "Planer hybrid systems," in Hybrid Systems II, ser. Lecture Notes in Computer Science 999, P. Antsaklis, A. Nerode, W. Kohn, and S. Sastry, Eds. Springer-Verlag, 1995, pp. 202-225.

[14] Y. Susuki, Y. Takatsuji, and T. Hikihara, "Hybrid model for cascading outage in a power system: A numerical study," (submitted).

[15] H. D. Chiang, C. C. Chu, and G. Cauley, "Direct stability analysis of electric power systems using energy functions: Theory, applications, and perspective," Proceedings of the IEEE, vol. 83, no. 11, pp. 1497-1529, November 1995.

[16] M. A. Pai, Energy Function Analysis for Power System Stability. Kluwer Academic Pub., 1989.

[17] L. G. Perez, A. J. Flechsig, and V. Venkatasubramanian, "Modeling the protective system for power system dynamic analysis," IEEE Transactions on Power Systems, vol. 9, no. 4, pp. 1963-1973, November 1994.

[18] T. A. Henzinger, "The theory of hybrid automata," in Proceedings of the 11th Annual IEEE Symposium on Logic in Computer Science, 1996, pp. $278-292$.

[19] I. M. Mitchell, "Comparing forward and backward reachability as tools for safety analysis," in Hybrid Systems: Computation and Control, ser. Lecture Notes in Computer Science 4416, A. Bemporad, A. Bicchi, and G. Buttazzo, Eds. Springer-Verlag, 2007, pp. 428-443. 